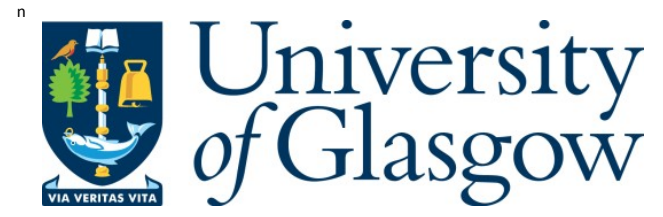

Wille, H., Ruess, M., Rank, E., and Y osibash, Z. (2016) Uncertainty quantification for personalized analyses of human proximal femurs. J ournal of Biomechanics.

There may be differences between this version and the published version. $Y$ ou are advised to consult the publisher's version if you wish to cite from it.

http://eprints.gla.ac.uk/116060/

Deposited on: 04 February 2016

Enlighten - R esearch publications by members of the U niversity of Glasgow http://eprints.gla.ac.uk 


\section{Author's Accepted Manuscript}

Uncertainty quantification for personalized analyses of human proximal femurs

Hagen Wille, Martin Ruess, Ernst Rank, Zohar Yosibash

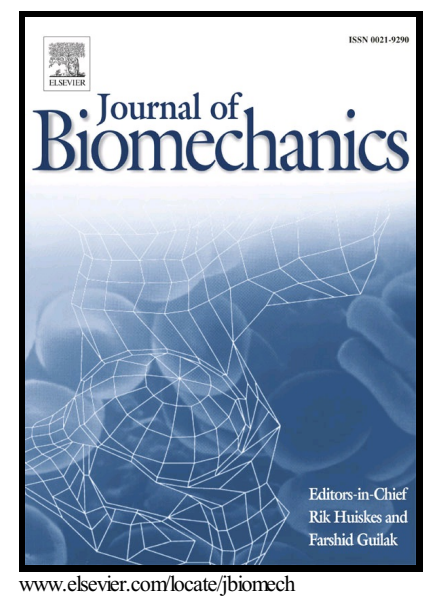

PII: $\quad$ S0021-9290(15)00643-0

DOI: $\quad$ http://dx.doi.org/10.1016/j.jbiomech.2015.11.013

Reference: BM7421

To appear in: Journal of Biomechanics

Received date: 23 January 2015

Revised date: 7 November 2015

Accepted date: 11 November 2015

Cite this article as: Hagen Wille, Martin Ruess, Ernst Rank and Zohar Yosibash Uncertainty quantification for personalized analyses of human proximal femurs Journal of Biomechanics, http://dx.doi.org/10.1016/j.jbiomech.2015.11.013

This is a PDF file of an unedited manuscript that has been accepted fo publication. As a service to our customers we are providing this early version o the manuscript. The manuscript will undergo copyediting, typesetting, an review of the resulting galley proof before it is published in its final citable form Please note that during the production process errors may be discovered whic could affect the content, and all legal disclaimers that apply to the journal pertain 


\title{
Uncertainty quantification for personalized analyses of human proximal femurs
}

\author{
Hagen Wille ${ }^{\mathrm{a}}$, Martin Ruess ${ }^{\mathrm{b}}$, Ernst Rank ${ }^{\mathrm{a}}$, Zohar Yosibash ${ }^{\mathrm{c}, *}$ \\ ${ }^{a}$ Chair for Computation in Engineering, Technische Universität München, Munich, Germany \\ ${ }^{b}$ Faculty of Aerospace Engineering, Delft University of Technology, Delft, Netherlands \\ ${ }^{c}$ Department of Mechanical Engineering, Ben-Gurion University of the Negev, Beer-Sheva, Israel
}

\begin{abstract}
Computational models for the personalized analysis of human femurs contain uncertainties in bone material properties and loads, which affect the simulation results. To quantify the influence we developed a probabilistic framework based on polynomial chaos (PC) that propagates stochastic input variables through any computational model. We considered a stochastic $E$ - $\rho$ relationship and a stochastic hip contact force, representing realistic variability of experimental data. Their influence on the prediction of principal strains $\left(\epsilon_{1}\right.$ and $\left.\epsilon_{3}\right)$ was quantified for one human proximal femur, including sensitivity and reliability analysis. Large variabilities in the principal strain predictions were found in the cortical shell of the femoral neck, with coefficients of variation of $\approx 40 \%$. Between $60-80 \%$ of the variance in $\epsilon_{1}$ and $\epsilon_{3}$ are attributable to the uncertainty in the $E$ - $\rho$ relationship, while $\approx 10 \%$ are caused by the load magnitude and 5-30\% by the load direction. Principal strain directions were unaffected by material and loading uncertainties. The antero-superior and medial inferior sides of the neck exhibited the largest probabilities for tensile and compression failure, however all were very small $\left(p_{f}<0.001\right)$. In summary, uncertainty quantification with PC has been demonstrated to efficiently and accurately describe the influence of very different stochastic inputs, which increases the credibility and explanatory power of personalized analyses of human proximal femurs.
\end{abstract}

Keywords: femur, personalized medicine, uncertainty quantification, polynomial chaos, finite cell method

\footnotetext{
${ }^{*}$ Corresponding Author

Email addresses: hagen.wille@tum.de (Hagen Wille), m.ruess@tudelft.nl (Martin Ruess), ernst.rank@tum.de (Ernst Rank), zohary@bgu.ac.il (Zohar Yosibash)
} 


\section{Introduction}

Computational models based on computed tomography $(\mathrm{CT})$ are widely used to predict the mechanical behavior of human femurs (Bessho et al., 2007, Yosibash et al., 2013, Schileo et al., 2014, Ali et al., 2014). These have demonstrated their predictive accuracy with respect to in-vitro experiments, where a well-defined load is applied (Schileo et al., 2007, Cristofolini et al., 2010, Trabelsi et al., 2011, Ruess et al., 2012). In clinical practice, however, personalized physiological loading conditions are required. Magnitude and direction of the hip contact force are usually inferred from in-vivo measurements (Bergmann et al., 2001, 2010), but contain uncertainties because of inter- and intra-patient variations. Another major challenge is the determination of heterogeneous material properties (Taddei et al., 2007, Helgason et al., 2008, Eberle et al., 2013). The large scatter in the experiments that determine the relationship between Young's modulus $E$ and a local densitometric measure $\rho$ also induces uncertainties. Quantifying the influence of both uncertainties on the predicted mechanical response of the femur is mandatory when advocating computational models for clinical practice.

Uncertainty quantification is an essential part of model validation and is performed in three steps: characterizing the uncertain parameters, propagating them through the computational model, and estimating the stochastic response of interest (Oberkampf et al., 2004). Probabilistic studies that performed uncertainty quantification for computational models of human femurs are summarized in Table 1 along with their stochastic components, probabilistic methods, and aim of research.

\section{[Table 1 about here.]}

Material and hip loading uncertainties have been characterized most frequently. Some studies assumed a random but homogeneous Young's modulus for the entire femur (Bah and Browne, 2009, Mehrez and Browne, 2012) or simplified the spatial distribution of cortical and trabecular bone (Chang et al., 2001, Nicolella et al., 2006, Viceconti et al., 2006). Others considered material uncertainties within the E- $\rho$ relationship (Chinchalkar and Taylor, 1989, 
Taddei et al., 2006, Laz et al., 2007, Long et al., 2009), but each study applied a different relationship with different random variables. Regarding the loading conditions, all studies considered a quasi-static loading representing walking free or going upstairs. Uncertainty in the hip contact force was commonly described with a random variable for the magnitude while the direction was assumed deterministic. Only few studies considered also uncertainties within the force direction (Nicolella et al., 2006, Long et al., 2009, Dopico-González et al., 2010). These conceptual differences led us to consider the stochastic $E$ - $\rho$ relationship from Wille et al. (2012) in combination with the stochastic description of the peak hip contact force magnitude and direction from Yosibash et al. (2015), both representing realistic variabilities of experimental data.

Various probabilistic methods have been used for propagating uncertainties through a computational model, among them Monte Carlo (MC) simulation (Taddei et al., 2006, Viceconti et al., 2006, Laz et al., 2007, Dopico-González et al., 2010, Mehrez and Browne, 2012), response surface methods (Chang et al., 2001, Bah and Browne, 2009, Long et al., 2009), and the advanced mean-value method (Nicolella et al., 2006, Laz et al., 2007). These methods have in common that they are non-intrusive with respect to the computational model, i.e. they use the computational model as a black box and require no access to the solver. Accuracy and efficiency of the probabilistic methods vary, depending on the number of uncertain parameters and the stochastic response of interest. In this study we employed a different probabilistic method based on the concept of polynomial chaos (PC) (Ghanem and Spanos, 1990, Xiu and Karniadakis, 2002, Xiu, 2009), which has been shown to be superior in many engineering problems over the past two decades.

Most studies in Table 1 investigated the influence of model uncertainties within the context of a total hip replacement, which increases the model complexity considerably. Only two studies analyzed the mechanical behavior of femurs without an implant (Taddei et al., 2006, Laz et al., 2007), but limited the investigation to global performance indicators such as the maximum von Mises stress. Results typically included some descriptive statistics of the response variable (e.g. mean, standard deviation, $1^{\text {st }}$ and $99^{\text {th }}$ percentile), estimates of its distribution (probability density function or cumulative distribution function), and/or sensitivity parameters and probabilities of failure. None of the studies quantified uncertainties 


\subsection{Stochastic input variables}

A stochastic relationship between ash density $\rho_{\text {ash }}$ and Young's modulus $E$ based on pooled data from multiple experimental studies on femur tissue was presented in Wille et al. 
(2012):

$$
E=12000 \cdot \rho_{\text {ash }}^{1.45} \cdot X_{E} \quad E \text { in }[\mathrm{MPa}], \rho_{\text {ash }} \text { in }\left[\mathrm{g} / \mathrm{cm}^{3}\right]
$$

1 with $X_{E} \sim \ln \mathcal{N}\left(\mu_{E}=0, \sigma_{E}^{2}=0.316^{2}\right)$ being a log-normal random variable. The two 2 parameters $\mu_{E}$ and $\sigma_{E}^{2}$ denote mean and variance of the associated normal distribution. $X_{E}$ 3 characterizes the scatter of residual values around the regression mean (Wille et al., 2012).

${ }_{4} \quad$ The stochastic loading model from Yosibash et al. (2015) was considered. It describes 5 variations in the peak hip contact force during walking free and going upstairs and was 6 derived from databases HIP98 (Bergmann, 2001) and OrthoLoad (Bergmann, 2008), which contain in-vivo measurements of the hip contact force (Bergmann et al., 2001, Heller et al., 8 2001). In total 141 data records from seven patients (3 female, 4 male, age: 55-82 y, weight: 49-101 kg) were considered for the stochastic loading model. Using the anatomical reference frame of OrthoLoad, the hip contact force is described by its magnitude $F$ and two corresponding angular directions $A_{x}$ and $A_{y}$; details are given in Yosibash et al. (2015).

We restricted this study to the stochastic loading model for going upstairs, as it has the largest spread in data:

$$
\begin{array}{rlrl}
X_{F} & \sim \mathcal{N}\left(0.97 \cdot B W+1465,277^{2}\right) & F, B W \text { in }[\mathrm{N}] \\
X_{A_{x}} & \sim \mathcal{N}\left(15.25,3.38^{2}\right) & & A_{x} \text { in }[\mathrm{deg}] \\
X_{A_{y}} & \sim \mathcal{N}\left(19.69,6.49^{2}\right) & & A_{y} \text { in }[\mathrm{deg}]
\end{array}
$$

where $B W$ denotes the body weight. The three independent normal random variables $X_{F}$, $X_{A_{x}}$, and $X_{A_{y}}$ characterize both inter- and intra-patient variability of the force magnitude and direction, respectively (Yosibash et al., 2015). Inserting the assumed body weight of $800 \mathrm{~N}$ in (2) yields $X_{F} \sim \mathcal{N}\left(2241,277^{2}\right)$, which completes the stochastic hip loading for the personalized analysis of bone FF5.

\subsection{Uncertainty propagation with polynomial chaos}

We consider the computational model as a black box denoted by $\mathcal{M}$ into which the random vector $\mathbf{X}=\left[X_{E}, X_{F}, X_{A_{x}}, X_{A_{y}}\right]^{T}$ is input, and denote the stochastic output of interest by $Y=\mathcal{M}(\mathbf{X})$. The random variable $Y$ can be any scalar quantity at a specific 
1 location, like maximum or minimum principal strains $\left(\epsilon_{1}\right.$ or $\left.\epsilon_{3}\right)$, or principal directions in 2 spherical coordinates (polar angle $\theta$ and azimuthal angle $\phi$ ).

The main idea of PC is to represent $Y$ by a series of orthogonal polynomials that depend on standardized random variables (Ghanem and Spanos, 1990). The distribution type of the input random variables defines the specific family of orthogonal polynomials. In our case, every input variable in $\mathbf{X}$ is related to an independent, standard normal random variable $U \sim \mathcal{N}(0,1):$

$$
\begin{gathered}
X_{E}=\exp \left(0.316 \cdot U_{1}\right) \\
X_{F}=2241+277 \cdot U_{2} \\
X_{A_{x}}=15.25+3.38 \cdot U_{3} \\
X_{A_{y}}=19.69+6.49 \cdot U_{4}
\end{gathered}
$$

3 for which the Hermite polynomials form an orthogonal basis (Xiu and Karniadakis, 2002).

${ }_{4} \quad$ The polynomial chaos expansion (PCE) of the stochastic response reads then:

$$
Y=\mathcal{M}(\mathbf{X})=\sum_{\alpha \in \mathcal{A}} y_{\alpha} \Psi_{\alpha}(\mathbf{U})
$$

${ }_{5}$ where $y_{\boldsymbol{\alpha}}$ are unknown coefficients, $\boldsymbol{\alpha}=\left(\alpha_{1}, \ldots, \alpha_{4}\right)$ a set of indices, and $\mathbf{U}=\left[U_{1}, U_{2}, U_{3}, U_{4}\right]^{T}$ 6 the vector of standard normal variables. The corresponding basis functions are $\Psi_{\alpha}(\mathbf{U})=$ $7 h_{\alpha_{1}}\left(U_{1}\right) \cdot h_{\alpha_{2}}\left(U_{2}\right) \cdot h_{\alpha_{3}}\left(U_{3}\right) \cdot h_{\alpha_{4}}\left(U_{4}\right)$ with $h_{\alpha_{i}}$ denoting the normalized Hermite polynomial 8 of degree $\alpha_{i}$. In practice, the PCE (9) is truncated at a specific order $p$ defining the highest 9 polynomial degree of $\Psi_{\alpha}$. This limits the total number of coefficients $y_{\alpha}$, which is given by the binomial coefficient $\left(\begin{array}{c}4+p \\ p\end{array}\right)$ (Sudret, 2008). When approximating $Y$ with PCEs of order $p=1,2,3$, or 4 , then only $5,15,35$, or 70 coefficients have to be determined, respectively.

Because the orthogonality of the basis functions is defined with respect to the expectation operator $\mathbb{E}$, it holds for the Hermite polynomials that:

$$
\mathbb{E}\left[\Psi_{\boldsymbol{\alpha}}(\mathbf{U}) \Psi_{\boldsymbol{\beta}}(\mathbf{U})\right]=\int_{\mathcal{D}_{\mathbf{U}}} \Psi_{\boldsymbol{\alpha}}(\boldsymbol{u}) \Psi_{\boldsymbol{\beta}}(\boldsymbol{u}) f_{\mathbf{U}}(\boldsymbol{u}) \mathrm{d} \boldsymbol{u}= \begin{cases}1 & \boldsymbol{\alpha}=\boldsymbol{\beta} \\ 0 & \boldsymbol{\alpha} \neq \boldsymbol{\beta}\end{cases}
$$

where $f_{\mathbf{U}}(\boldsymbol{u})$ is the joint probability density function of random vector $\mathbf{U}$, which defines for every event $\boldsymbol{u}$ in the support space $\mathcal{D}_{\mathbf{U}}$ the respective probability measure $f_{\mathbf{U}}(\boldsymbol{u}) \mathrm{d} \boldsymbol{u}$. 
1 Utilizing (10) in combination with (9), the coefficients $y_{\boldsymbol{\alpha}}$ are obtained by:

$$
y_{\boldsymbol{\alpha}}=\mathbb{E}\left[Y \Psi_{\boldsymbol{\alpha}}(\mathbf{U})\right]=\int_{\mathcal{D}_{\mathbf{U}}} \mathcal{M}(\boldsymbol{x}) \Psi_{\boldsymbol{\alpha}}(\boldsymbol{u}) f_{\mathbf{U}}(\boldsymbol{u}) \mathrm{d} \boldsymbol{u}
$$

2

4

5

The integral (11) is approximated by a quadrature:

$$
y_{\boldsymbol{\alpha}} \approx \sum_{i=1}^{Z} w_{i} \mathcal{M}\left(\boldsymbol{x}^{(i)}\right) \Psi_{\boldsymbol{\alpha}}\left(\boldsymbol{u}^{(i)}\right)
$$

with $\boldsymbol{u}^{(i)}$ and $w_{i}$ denoting the quadrature points and weights of the Gauss-Hermite quadrature, respectively. The corresponding model inputs $\boldsymbol{x}^{(i)}$ are derived from $\boldsymbol{u}^{(i)}$ using (5)-(8). Evaluating the multi-dimensional integral (11) by tensor products of $1-\mathrm{D}$ quadrature results in an exponential growth of quadrature points as the number of dimensions increases.

This curse of dimensionality is circumvented by using Smolyak's quadrature scheme instead (Smolyak, 1963); we utilized the implementation provided by Heiss and Winschel (2008).

When $Y$ is approximated with a PCE of order $p=1,2,3$, or 4 for instance, then the computation of all 5, 15, 35, or 70 coefficients with (12) requires only 9, 41, 137, or 385 simulation runs with the model $\mathcal{M}$, respectively. Each simulation run evaluates then a different combination of $E$ - $\rho$ relationship, force magnitude, and load directions, according to the quadrature points. The hierarchical nature of both PCEs and Smolyak's quadrature scheme allowed us to adaptively increase the number of quadrature points and to reuse lower order approximations.

A MC simulation was conducted in order to verify the results of the PCE. MC involves repeated random sampling of $\mathbf{X}$ and solving for each a deterministic problem, which results in a sample set of $Y$. From this we computed the sample variance and an estimate of the response distribution (histogram). Due to the slow convergence rate of the MC simulation, many deterministic simulation runs are required; we performed 10000.

\subsection{Post-processing the stochastic response}

After all coefficients have been determined using (12), the mean $\mu_{Y}$ of the stochastic response $Y$ is described by the coefficient $y_{\mathbf{0}}$, and the variance $\sigma_{Y}^{2}$ of the stochastic response 
is the sum of squares of all coefficients except $y_{\mathbf{0}}$ (Sudret, 2008):

$$
\begin{aligned}
\mu_{Y} & =\mathbb{E}[Y]=y_{0} \\
\sigma_{Y}^{2} & =\mathbb{E}\left[\left(Y-\mu_{Y}\right)^{2}\right]=\sum_{\boldsymbol{\alpha} \in \mathcal{A} \backslash \mathbf{0}} y_{\boldsymbol{\alpha}}^{2}
\end{aligned}
$$

Please note that both parameters do not imply that $Y$ is normally distributed, but are two parameters that describe location and dispersion, respectively, of any response distribution. We obtained the probability density function (PDF) of $Y$ by kernel smoothing a large set of response samples (Wand and Jones, 1995). For this purpose, the PCE was used as a surrogate model to generate $10^{6}$ samples of the response variable. These samples were also used for a reliability analysis. For that we considered a simple strain based failure criterion (Schileo et al., 2008, Yosibash et al., 2010, Schileo et al., 2014), which uses the yield strain for femoral bone tissue in tension $\epsilon_{\lim , T}=7300 \mu \mathrm{m} / \mathrm{m}$ and compression $\epsilon_{\lim , C}=-10400 \mu \mathrm{m} / \mathrm{m}$ (Bayraktar et al., 2004) as threshold values. Consequently, we defined the failure probability $p_{f}$ for (local) tensile or compression failure as the likelihood that the maximum principal strain $\epsilon_{1} \geq \epsilon_{\lim , T}$ or the minimum principal strain $\epsilon_{3} \leq \epsilon_{\lim , C}$. We approximated $p_{f}$ from the number of response samples fulfilling the respective criteria, divided by the total number of samples.

PCEs can be also used for a global sensitivity analysis. Sudret (2008) proved that PCEs are identical to Sobol' decompositions of the model $\mathcal{M}$ (Sobol', 2001) and that the corresponding Sobol' indices can be computed directly from the coefficients of the PCE. Sobol' indices are global sensitivity indices, which represent the fraction of the response variance that can be attributed to a specific input variable or their interactions. The first-order sensitivity indices $S_{E}, S_{F}, S_{A_{x}}$, and $S_{A_{y}}$ quantify in percentage the influence of each input variable taken alone and were computed from the square-summed and normalized coefficients that are exclusively associated with the respective input variable (Sudret, 2008). Also the sum of all remaining higher-order interaction indices was computed using $\sum S_{i j}=1-S_{E}-S_{F}-S_{A_{x}}-S_{A_{y}}$.

Finally, the stochastic results were visualized pointwise for an intuitive interpretation. We depicted mean and variance of the maximum and minimum principal strain with a sphere whose color is defined by the mean, whereas the radius depends on the standard deviation. Failure probabilities were also represented by colored spheres, however all radii 
1 were identical in that case. We verified the stochastic results of the PCE by comparing the

$\mathrm{PDF}$ with the respective histogram of the MC simulation. Moreover, we investigated the convergence behavior of both methods approximating the variance.

\subsection{Deterministic simulation by FCM}

The FCM embeds the voxel-based geometry of bone FF5 in a simulation domain of hexahedral cells following a regular Cartesian grid. Figure 1 illustrates this concept, which omits a computational expensive segmentation and meshing procedure, as required for standard finite element analyses. The FCM proved to be highly efficient for linear elastic analyses of bones and was validated by in-vitro experiments in a previous study (Ruess et al., 2012).

[Figure 1 about here.]

The large computational cells were implemented as p-version hexahedral elements (Düster et al., 2008) and should not be mistaken for low order finite elements. Within one finite cell material properties can vary significantly, and it was shown that a coarse grid of cells is sufficient to obtain accurate results for a fine voxel resolution like the one used in the present study (Ruess et al., 2012). Inhomogeneous isotropic material properties were assumed for bone tissue (Trabelsi et al., 2011). After converting Hounsfield unit $H U$ into equivalent mineral density $\rho_{\text {eqm }}$ and then into ash density $\rho_{\text {ash }}$ based on:

$$
\begin{array}{ll}
\rho_{\text {eqm }}=10^{-3} \cdot(0.793 \cdot H U+4.183) & {\left[\mathrm{g} / \mathrm{cm}^{3}\right]} \\
\rho_{\text {ash }}=1.22 \cdot \rho_{\text {eqm }}+0.0523 & {\left[\mathrm{~g} / \mathrm{cm}^{3}\right]}
\end{array}
$$

where (15) is the calibration of the CT-scan with $\mathrm{K}_{2} \mathrm{HPO}_{4}$ phantoms and (16) is from Keyak and Falkinstein (2003), the heterogeneous Young's modulus was derived from (1). Because the random variable in (1) is location independent, it affects the heterogeneous Young's modulus everywhere the same and thus can be regarded as a global scaling factor. In all computations a constant Poisson ratio $\nu=0.3$ was used.

The hip contact force was modeled as a surface load, which distributes the force over a locally confined contact area on the head of the femur. Since the head is approximately spherical, the contact area was designed as a spherical cap (radius of sphere: $24 \mathrm{~mm}$, radius 
of base of the cap: $10 \mathrm{~mm}$ ). The applied pressure load and the orientation of the contact area were chosen according to the stochastic input variables $X_{F}, X_{A_{x}}$ and $X_{A_{y}}$.

Within the probabilistic framework this computational model was used to assess maximum and minimum principal strains $\left(\epsilon_{1}, \epsilon_{3}\right)$ and principal directions $(\theta, \phi)$ at 884 postprocessing locations (554 spread along the femoral cortex, 330 uniformly filling the trabecular compartment and the diaphysis).

\subsection{Verifying FCM's accuracy}

Prior to uncertainty quantification, the accuracy of the deterministic computational model was rechecked by comparison to an in-vitro experiment (Yosibash et al., 2013). The femur was loaded as in the experiment $\left(F=1000 \mathrm{~N}, A_{x}=A_{y}=0 \mathrm{deg}\right)$, and the median of the stochastic $E$ - $\rho$ relationship (1) was used, since Median $\left(X_{E}\right)=1$. Principal strains were computed for the 11 surface locations (four at the superior and inferior neck, seven at the medial and lateral diaphysis) at which strains had been measured (Yosibash et al., 2013, Fig 2). Convergence in energy norm was investigated by increasing the polynomial order of the $\mathrm{FCM}$, i.e. $p_{\mathrm{FCM}}=1, \ldots, 5$.

\section{Results}

The deterministic FCM has a high predictive accuracy: computed strains matched well the ones measured in the experiment (correlation $r=0.987$, average relative error of $18 \%$ ). Convergence in energy norm was achieved for $p_{\mathrm{FCM}}=4$ (with an error of $8.4 \%$ ). Thus, all simulation runs were performed with $p_{\mathrm{FCM}}=4$.

The stochastic results were verified by comparing the PDFs of the PCE to the respective histograms of the MC simulation, as depicted exemplarily for one post-processing location in Figure 2. The PCE of order $p=4$ (385 Smolyak runs) was in excellent agreement with the MC simulation (10000 runs), which is also reflected in the convergence behavior of both methods (Figure 2b).

[Figure 2 about here.]

Means and standard deviations of the maximum and minimum principal strains are shown in Figure 3. The vast majority of large strain values were found within the cortical shell. 
Given that the loading had a pronounced inclination in the sagittal plane, $\epsilon_{1}$ was largest at the anterior side at the distal diaphysis $(\mu=2380, \sigma=955)$ and the antero-superior neck $(\mu=1569, \sigma=656)$. Contrarily, the largest values for $\epsilon_{3}$ were predominantly at the posterior side of the femur, with strain concentrations at the distal diaphysis $(\mu=-3230$, $\sigma=1270)$ and the medial neck above the lesser trochanter $(\mu=-2296, \sigma=824)$.

[Figure 3 about here.]

The largest failure probabilities within the region of the femoral neck were found to be $p_{f}=13 \cdot 10^{-6}$ for tensile failure (point 351 in Figure $4 \mathrm{a}$ ) and $p_{f}=2 \cdot 10^{-6}$ for compression failure (point 231 in Figure 4b), respectively. Both locations are in the cortical shell. In general all failure probabilities were small $\left(p_{f}<0.001\right.$, at many locations even $\left.p_{f}<10^{-6}\right)$.

[Figure 4 about here.]

Results of the sensitivity analysis for these two points are summarized in Table 2. $\epsilon_{1}$ and $\epsilon_{3}$ were most sensitive to the stochastic $E-\rho$ relationship $\left(S_{E} \approx 60 \%\right.$ for $\epsilon_{1}$ at point 351 , and $S_{E} \approx 80 \%$ for $\epsilon_{3}$ at point 231 ). Around $10 \%$ of the variance in the principal strain magnitudes is attributed to the force magnitude; the influence of the load angles is larger for $\epsilon_{1}\left(S_{A_{x}}=15.2 \%, S_{A_{y}}=12.2 \%\right)$ than for $\epsilon_{3}\left(S_{A_{x}}=2.8 \%, S_{A_{y}}=2.4 \%\right)$. Different results were obtained for the principal directions: $\theta$ and $\phi$ of both principal strains were affected solely by $A_{x}$ and $A_{y}$, which accounted together for more than $95 \%$ of the respective variances. The $E-\rho$ relationship and the force magnitude had no influence on the principal directions $\left(S_{E}, S_{F}<10^{-6}\right)$. Note that the standard deviation of $\theta$ and $\phi$ were found to be very small $(<5 \mathrm{deg})$. Although the load angles explained the vast majority of the variance, their absolute effect on the principal directions is minor.

[Table 2 about here.]

\section{Discussion}

A probabilistic framework was developed to quantify the influence of material and loading uncertainties on the prediction of principal strains within a personalized analysis of a 
1 human proximal femur. The predicted strains were highly affected by the four stochastic input variables, which represented experimental variabilities in the $E$ - $\rho$ relationship and the hip contact force. The large standard deviations for $\epsilon_{1}(\sigma \leq 656)$ and $\epsilon_{3}(\sigma \leq 824)$ at the femoral neck imply broad probability distributions and thus large ranges of possible strain predictions. Even broader distributions were found at the distal diaphysis but can be attributed to the clamped boundary condition. The coefficient of variation (standard deviation divided by the mean) was $\approx 40 \%$ for both $\epsilon_{1}$ and $\epsilon_{3}$ at all locations with sufficiently large mean values. This relative variability within the principal strain predictions is larger than the one reported in Taddei et al. (2006), which computed coefficients of variation of less than $9 \%$ for $\max \epsilon_{1}$ and $\max \epsilon_{3}$. These differences may be explained by the uncertainty in the $E$ 1 relationship, which is larger in the current study. For comparison purposes, assuming a 12 bone density of $\rho_{\text {ash }}=1.2 \mathrm{~g} / \mathrm{cm}^{3}$, the $95 \%$ prediction band of the Young's modulus ranged between 8.4-29.1 GPa in this study, compared to 16.6-16.9 GPa in Taddei et al. (2006).

The global sensitivity analysis corroborated the dominating influence of the stochastic $E$ - $\rho$ relationship on the magnitude of $\epsilon_{1}$ and $\epsilon_{3}$. Within the femoral neck, between 60$80 \%$ of the variance of both principal strains were explained by the uncertainty in the $E-\rho$ relationship. Because of its large influence compared to the other three input variables, a reduction of the uncertainty in the $E$ - $\rho$ relationship would have the largest effect on $\epsilon_{1}$ and $\epsilon_{3}$ and narrow the response distributions the most. This would require new experiments on bone tissue specimens with a well defined protocol that reduces the large spread in the current experimental data (Helgason et al., 2008, Wille et al., 2012).

Predictions of the principal strain direction, on the other hand, were completely unaffected by the uncertainties in the $E$ - $\rho$ relationship and the force magnitude. Only the load direction had an influence on the direction of the principal strains. However, the effect was marginal as the standard deviation of the principal strain directions was found to be very small within the femoral neck $(<5 \mathrm{deg})$, which is in agreement with experimental observations. Cristofolini et al. (2009) measured strains in 24 femurs for six different loading configurations and reported for all strain measurements a standard deviation of $6.7 \mathrm{deg}$.

A comparison with sensitivity results from other stochastic studies (Table 1) renders difficult. The main reason is that different sensitivity measures were used. Some studies 
reported Pearson's correlation coefficient between the stochastic input variables and the response variables as sensitivity indices (Taddei et al., 2006, Viceconti et al., 2006, DopicoGonzález et al., 2010), which assess the strength of the linear association between them. In case the input affects non-linearly the computational model, such a correlation analysis can be very misleading. More meaningful are local sensitivity analyses, with sensitivity indices measuring how changes in the parameters of the stochastic input variables (e.g. mean or standard deviation) affect the probability of failure (Nicolella et al., 2006, Mehrez and Browne, 2012). In contrast, we performed a global sensitivity analysis, which is based on a variance decomposition for the stochastic response. Here, sensitivity indices quantify the percentage of the response variance attributable to an entire input variable or combinations of variables.

Regarding the reliability analysis, all failure probabilities were found to be very small $\left(p_{f}<0.001\right)$. The antero-superior neck region of this femur is more likely to suffer from local tension failure $\left(p_{f} \leq 13 \cdot 10^{-6}\right)$ than the postero-medial neck region from compression failure $\left(p_{f} \leq 2 \cdot 10^{-6}\right)$, when loaded with the peak hip contact force during going upstairs. However, both events are extremely improbable; an expected outcome given that the subject had no skeletal disease. Note that these local failure probabilities are not implying any global clinical failure or bone fracture. Instead they describe the likelihood of irreversible damage in the bone tissue at the respective location, which may cause fracture initiation.

The presented uncertainty quantification, including sensitivity and reliability analysis, was used largely to demonstrate the probabilistic framework based on PC. This powerful and widely accepted approach is novel in biomechanics, and to the best of our knowledge was only used for a cardiovascular simulation (Sankaran and Marsden, 2011). The PC approach was two orders of magnitude computationally more efficient than an extensive MC simulation (10000 runs) performed to verify the stochastic results. In fact, a PCE of lower order $(p=3)$ would have been sufficient to approximate the stochastic response in this study (cf. Figure 2), further reducing the number of necessary simulation runs from 385 to 137 . Uncertainty quantification with a specific objective, e.g. determining percentiles or computing failure probabilities, might be possible at even lower computational costs with the advanced mean value method (Nicolella et al., 2006, Laz et al., 2007). However, a meaningful 
comparison would require both methods to be applied to the same stochastic problem.

Further limitations of this study are related to the computational model and the stochastic input variables. Any personalized computational model requires assumptions on the geometry, material behavior, and boundary conditions. Uncertainties in geometry due to imprecise segmentation from CT-scans were not addressed, because they are considered negligible compared to material and loading uncertainties (Gelaude et al., 2008, Trabelsi et al., 2009). The material behavior of the bone was assumed to be linear elastic and isotropic, which is a simplification of the reality that proved to be reasonable for stance-like loading conditions (Trabelsi et al., 2011). Orthotropic or transversely isotropic material models may become necessary in case of loading conditions that introduce considerable torsional moments, e.g. during sidewise falling. Another limitation is the clamped boundary condition at the distal diaphysis. Although mean deflections were within physiological range $(<2.5 \mathrm{~mm})$, realistic physiological boundary conditions would include kinematic constraints at the joints as well as muscle forces (Speirs et al., 2007). The absence of muscle forces in this study explains the very small strains observed in the greater trochanter area. However, we are unaware of any (stochastic) muscle force model that is suitable for the personalized analysis of human femurs. Clearly, all limitations related to the stochastic input variables pass on to the probabilistic analysis. For example, the stochastic peak hip contact force was derived from an elderly population (age 55-82 y) with total hip replacements (Yosibash et al., 2015). Here, the subject (age $56 \mathrm{y}$ ) falls inside the range, but had no hip implant. Moreover, the stochastic $E-\rho$ relationship was obtained by pooling data sets of various experimental studies (Wille et al., 2012). The different experimental protocols contributed considerably to the uncertainty in the $E$ - $\rho$ relationship. In both cases it is important to enhance the respective data basis with further experimental results, as this will improve the description of the stochastic input parameters.

In closing, a probabilistic framework that allows to quantify the influence of parameter uncertainties on the personalized analysis of human femurs was presented. The probabilistic framework can be applied to any computational model and easily extended by additional stochastic input variables. Future studies may not only perform uncertainty quantification for the prediction of principal strains, but investigate also displacements, stresses, global 
1 bone stiffness, implant micro-motions etc. The global sensitivity indices can then be used

\section{Acknowledgments} TUM for helpful discussions.

\section{References} Biomechanics 47, 536-543.

\section{Conflict of interest statement}

None of the authors have any conflict of interest to declare that could prejudice this work.

The authors thank the anonymous reviewers for their valuable and constructive comments leading to improvements in the content and presentation. We acknowledge the generous support of the Institute for Advanced Study of the Technische Universität München (TUM), funded by the German Excellence Initiative, and thank Dr. Iason Papaioannou from the

Ali, A.A., Cristofolini, L., Schileo, E., Hu, H., Taddei, F., Kim, R.H., Rullkoetter, P.J., Laz, P.J., 2014. Specimen-specific modeling of hip fracture pattern and repair. Journal of

Bah, M.T., Browne, M., 2009. Effect of geometrical uncertainty on cemented hip implant structural integrity. Journal of Biomechanical Engineering 131, 054501.

Bayraktar, H.H., Morgan, E.F., Niebur, G.L., Morris, G.E., Wong, E.K., Keaveny, T.M., 2004. Comparison of the elastic and yield properties of human femoral trabecular and cortical bone tissue. Journal of Biomechanics 37, 27-35.

Bergmann, G. (Ed.), 2001. HIP98 - Loading of the Hip Joint. Julius Wolff Institute, Charité - Universitätsmedizin Berlin. www.OrthoLoad.com. 
1 Bergmann, G. (Ed.), 2008. OrthoLoad database. Charité - Universitätsmedizin Berlin. www.OrthoLoad.com.

Bergmann, G., Deuretzbacher, G., Heller, M.O., Graichen, F., Rohlmann, A., Strauss, J., Duda, G.N., 2001. Hip contact forces and gait patterns from routine activities. Journal of Biomechanics 34, 859-871.

Bergmann, G., Graichen, F., Rohlmann, A., Bender, A., Heinlein, B., Duda, G.N., Heller, M.O., Morlock, M.M., 2010. Realistic loads for testing hip implants. Bio-Medical Materials and Engineering 20, 65-75.

Bessho, M., Ohnishi, I., Matsuyama, J., Matsumoto, T., Imai, K., Nakamura, K., 2007. Prediction of strength and strain of the proximal femur by a CT-based finite element method. Journal of Biomechanics 40, 1745-1753.

Chang, P.B., Williams, B.J., Bhalla, K.S.B., Belknap, T.W., Santner, T.J., Notz, W.I., Bartel, D.L., 2001. Design and Analysis of Robust Total Joint Replacements: Finite Element Model Experiments With Environmental Variables. Journal of Biomechanical Engineering 123, 239-246.

Chinchalkar, S., Taylor, D.L., 1989. Loading and Material Property Uncertainties in Finite Element Analysis for Orthopedics, in: Thirty-fifth Conference on the Design of Experiments, U.S. Army Research Office, Monterey, USA, 13-20 October 1989. pp. 91-102.

Cristofolini, L., Juszczyk, M.M., Taddei, F., Viceconti, M., 2009. Strain distribution in the proximal human femoral metaphysis. Proceedings of the Institution of Mechanical Engineers, Part H: Journal of Engineering in Medicine 223, 273-288.

Cristofolini, L., Schileo, E., Juszczyk, M.M., Taddei, F., Martelli, S., Viceconti, M., 2010. Mechanical testing of bones: the positive synergy of finite-element models and in vitro experiments. Philosophical transactions. Series A, Mathematical, physical, and engineering sciences $368,2725-2763$.

Dopico-González, C., New, A.M.R., Browne, M., 2010. Probabilistic finite element analysis 
of the uncemented hip replacement-effect of femur characteristics and implant design geometry. Journal of Biomechanics 43, 512-520.

Düster, A., Parvizian, J., Yang, Z., Rank, E., 2008. The finite cell method for threedimensional problems of solid mechanics. Computer Methods in Applied Mechanics and Engineering 197, 3768-3782.

Eberle, S., Göttlinger, M., Augat, P., 2013. Individual density-elasticity relationships improve accuracy of subject-specific finite element models of human femurs. Journal of Biomechanics 46, 2152-2157.

Gelaude, F., Vander Sloten, J., Lauwers, B., 2008. Accuracy assessment of CT-based outer surface femur meshes. Computer Aided Surgery 13, 188-199.

Ghanem, R.G., Spanos, P.D., 1990. Polynomial Chaos in Stochastic Finite Elements. Journal of Applied Mechanics 57, 197.

Grasa, J., Pérez, M.A., Bea, J.A., García-Aznar, J.M., Doblaré, M., 2005. A probabilistic damage model for acrylic cements. Application to the life prediction of cemented hip implants. International Journal of Fatigue 27, 891-904.

Heiss, F., Winschel, V., 2008. Likelihood approximation by numerical integration on sparse grids. Journal of Econometrics 144, 62-80.

Helgason, B., Perilli, E., Schileo, E., Taddei, F., Brynjólfsson, S., Viceconti, M., 2008. Mathematical relationships between bone density and mechanical properties: a literature review. Clinical Biomechanics 23, 135-146.

Heller, M.O., Bergmann, G., Deuretzbacher, G., Dürselen, L., Pohl, M., Claes, L., Haas, N.P., Duda, G.N., 2001. Musculo-skeletal loading conditions at the hip during walking and stair climbing. Journal of Biomechanics 34, 883-893.

Keyak, J.H., Falkinstein, Y., 2003. Comparison of in situ and in vitro CT scan-based finite element model predictions of proximal femoral fracture load. Medical Engineering \& Physics 25, 781-787. 
Laz, P.J., Stowe, J.Q., Baldwin, M.A., Petrella, A.J., Rullkoetter, P.J., 2007. Incorporating uncertainty in mechanical properties for finite element-based evaluation of bone mechanics. Journal of Biomechanics 40, 2831-2836.

Long, J.P., Santner, T.J., Bartel, D.L., 2009. Hip resurfacing increases bone strains associated with short-term femoral neck fracture. Journal of Orthopaedic Research 27, 1319-25.

Mehrez, L., Browne, M., 2012. A numerically validated probabilistic model of a simplified total hip replacement construct. Computer Methods in Biomechanics and Biomedical Engineering 15, 845-858.

Nicolella, D.P., Thacker, B.H., Katoozian, H., Davy, D.T., 2006. The effect of threedimensional shape optimization on the probabilistic response of a cemented femoral hip prosthesis. Journal of Biomechanics 39, 1265-1278.

Oberkampf, W.L., Trucano, T.G., Hirsch, C., 2004. Verification, validation, and predictive capability in computational engineering and physics. Applied Mechanics Reviews 57, 345.

Pérez, M.A., Grasa, J., García-Aznar, J.M., Bea, J.A., Doblaré, M., 2006. Probabilistic analysis of the influence of the bonding degree of the stem-cement interface in the performance of cemented hip prostheses. Journal of Biomechanics 39, 1859-1872.

Ruess, M., Tal, D., Trabelsi, N., Yosibash, Z., Rank, E., 2012. The finite cell method for bone simulations: verification and validation. Biomechanics and Modeling in Mechanobiology $11,425-437$.

Sankaran, S., Marsden, A.L., 2011. A stochastic collocation method for uncertainty quantification and propagation in cardiovascular simulations. Journal of Biomechanical Engineering 133, 031001.

Schileo, E., Balistreri, L., Grassi, L., Cristofolini, L., Taddei, F., 2014. To what extent can linear finite element models of human femora predict failure under stance and fall loading configurations? Journal of Biomechanics 47, 3531-3538. 
Schileo, E., Taddei, F., Cristofolini, L., Viceconti, M., 2008. Subject-specific finite element models implementing a maximum principal strain criterion are able to estimate failure risk and fracture location on human femurs tested in vitro. Journal of Biomechanics 41, $356-367$.

Schileo, E., Taddei, F., Malandrino, A., Cristofolini, L., Viceconti, M., 2007. Subjectspecific finite element models can accurately predict strain levels in long bones. Journal of Biomechanics 40, 2982-2989.

Smolyak, S., 1963. Quadrature and interpolation formulas for tensor products of certain classes of functions. Soviet Mathematics Doklady 4.

Sobol', I., 2001. Global sensitivity indices for nonlinear mathematical models and their Monte Carlo estimates. Mathematics and Computers in Simulation 55, 271-280.

Speirs, A.D., Heller, M.O., Duda, G.N., Taylor, W.R., 2007. Physiologically based boundary conditions in finite element modelling. Journal of Biomechanics 40, 2318-2323.

Sudret, B., 2008. Global sensitivity analysis using polynomial chaos expansions. Reliability Engineering \& System Safety 93, 964-979.

Taddei, F., Martelli, S., Reggiani, B., Cristofolini, L., Viceconti, M., 2006. Finite-element modeling of bones from CT data: sensitivity to geometry and material uncertainties. IEEE Transactions on Biomedical Engineering 53, 2194-2200.

Taddei, F., Schileo, E., Helgason, B., Cristofolini, L., Viceconti, M., 2007. The material mapping strategy influences the accuracy of CT-based finite element models of bones: an evaluation against experimental measurements. Medical Engineering \& Physics 29, 973-979.

Trabelsi, N., Yosibash, Z., Milgrom, C., 2009. Validation of subject-specific automated p-FE analysis of the proximal femur. Journal of Biomechanics 42, 234-241.

Trabelsi, N., Yosibash, Z., Wutte, C., Augat, P., Eberle, S., 2011. Patient-specific finite element analysis of the human femur-a double-blinded biomechanical validation. Journal of Biomechanics 44, 1666-1672. 
1 Viceconti, M., Brusi, G., Pancanti, A., Cristofolini, L., 2006. Primary stability of an anatom-

2 ical cementless hip stem: a statistical analysis. Journal of Biomechanics 39, 1169-1179.

3 Wand, M.P., Jones, M.C., 1995. Kernel Smoothing. Chapman \& Hall/CRC Monographs on

$4 \quad$ Statistics \& Applied Probability. 1. ed. ed., Chapman \& Hall, London.

${ }_{5}$ Wille, H., Rank, E., Yosibash, Z., 2012. Prediction of the mechanical response of the femur

$6 \quad$ with uncertain elastic properties. Journal of Biomechanics 45, 1140-1148.

7 Xiu, D., 2009. Fast numerical methods for stochastic computations: a review. Communica$8 \quad$ tions in Computational Physics 5, 242-272.

9 Xiu, D., Karniadakis, G.E., 2002. The Wiener-Askey Polynomial Chaos for Stochastic Differential Equations. SIAM Journal on Scientific Computing 24, 619-644.

Yosibash, Z., Katz, A., Milgrom, C., 2013. Toward verified and validated FE simulations of a femur with a cemented hip prosthesis. Medical Engineering \& Physics 35, 978-987.

Yosibash, Z., Tal, D., Trabelsi, N., 2010. Predicting the yield of the proximal femur using high-order finite-element analysis with inhomogeneous orthotropic material properties. Philosophical transactions. Series A, Mathematical, physical, and engineering sciences $368,2707-2723$.

Yosibash, Z., Wille, H., Rank, E., 2015. Stochastic description of the peak hip contact force during walking free and going upstairs. Journal of Biomechanics 48, 1015-1022. 


\section{Tables}

Table 1: Summary of probabilistic studies performing uncertainty quantification for computational models of human femurs. The checkmark symbol $(\checkmark)$ indicates which components of the femur model were considered to be stochastic. 
Table 2: Global sensitivity analysis for $\epsilon_{1}$ at point 351 and $\epsilon_{3}$ at point 231 (magnitude and $\theta$ and $\phi$ of the corresponding eigenvector). Shown are mean $\mu$, standard deviation $\sigma$, related first-order sensitivity indices $S_{F}, S_{A_{x}}, S_{A_{y}}, S_{E}$, and the sum of all remaining higher-order interaction indices $S_{i j}$. 

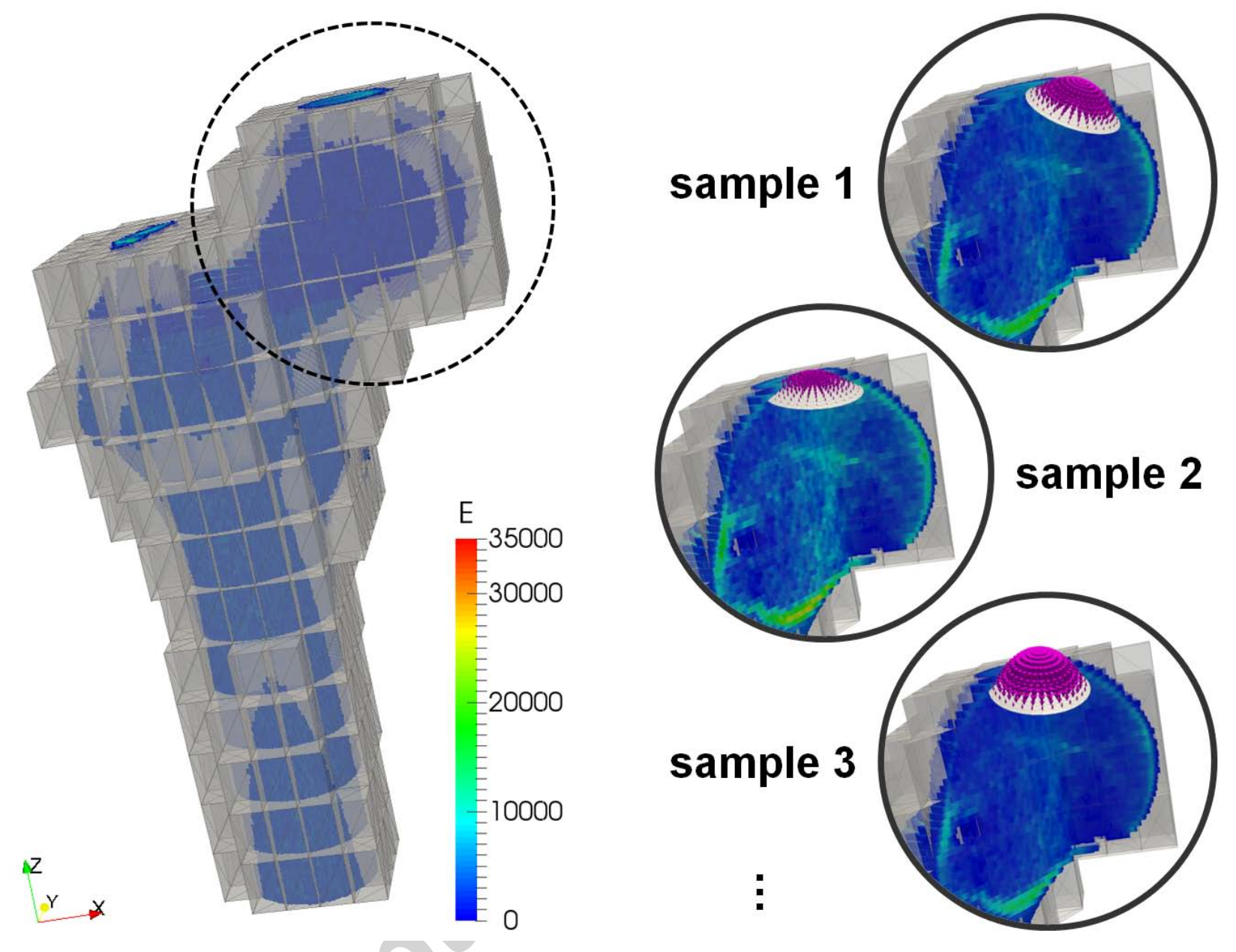

Figure 1: Deterministic finite cell model based on the CT scan of bone FF5 reported in Yosibash et al. (2013). The computational model is clamped at the distal end of the diaphysis. The hip contact force is modeled as surface load on a confined contact area on the head. During uncertainty quantification the heterogeneous Young's modulus $E$ and the surface load (magnitude and orientation) are changed for every simulation run, as depicted by the samples that give an insight into the region of the dashed circle. 


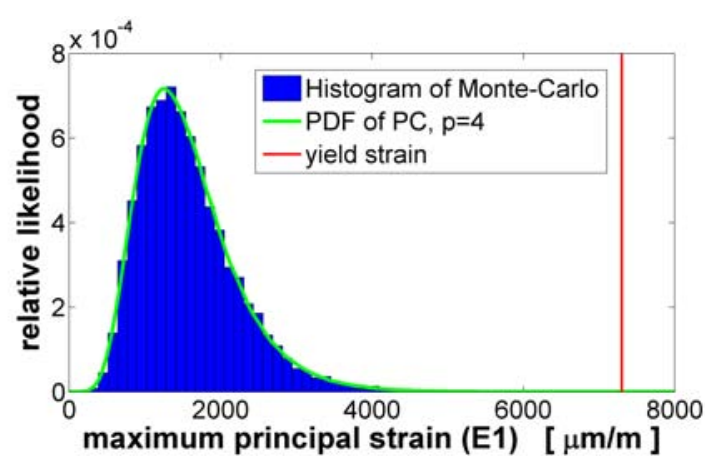

(a) Probability distribution of $\epsilon_{1}$

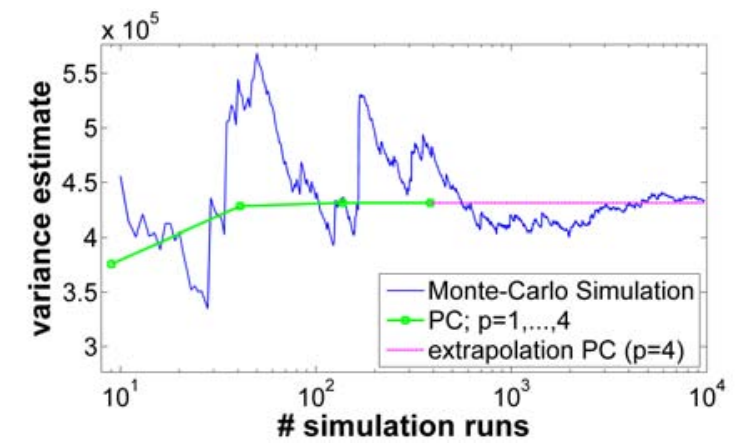

(b) Convergence plot for estimating the variance of $\epsilon_{1}$

Figure 2: Comparison of PCE with MC simulation for $\epsilon_{1}$ at post-processing point 351. 

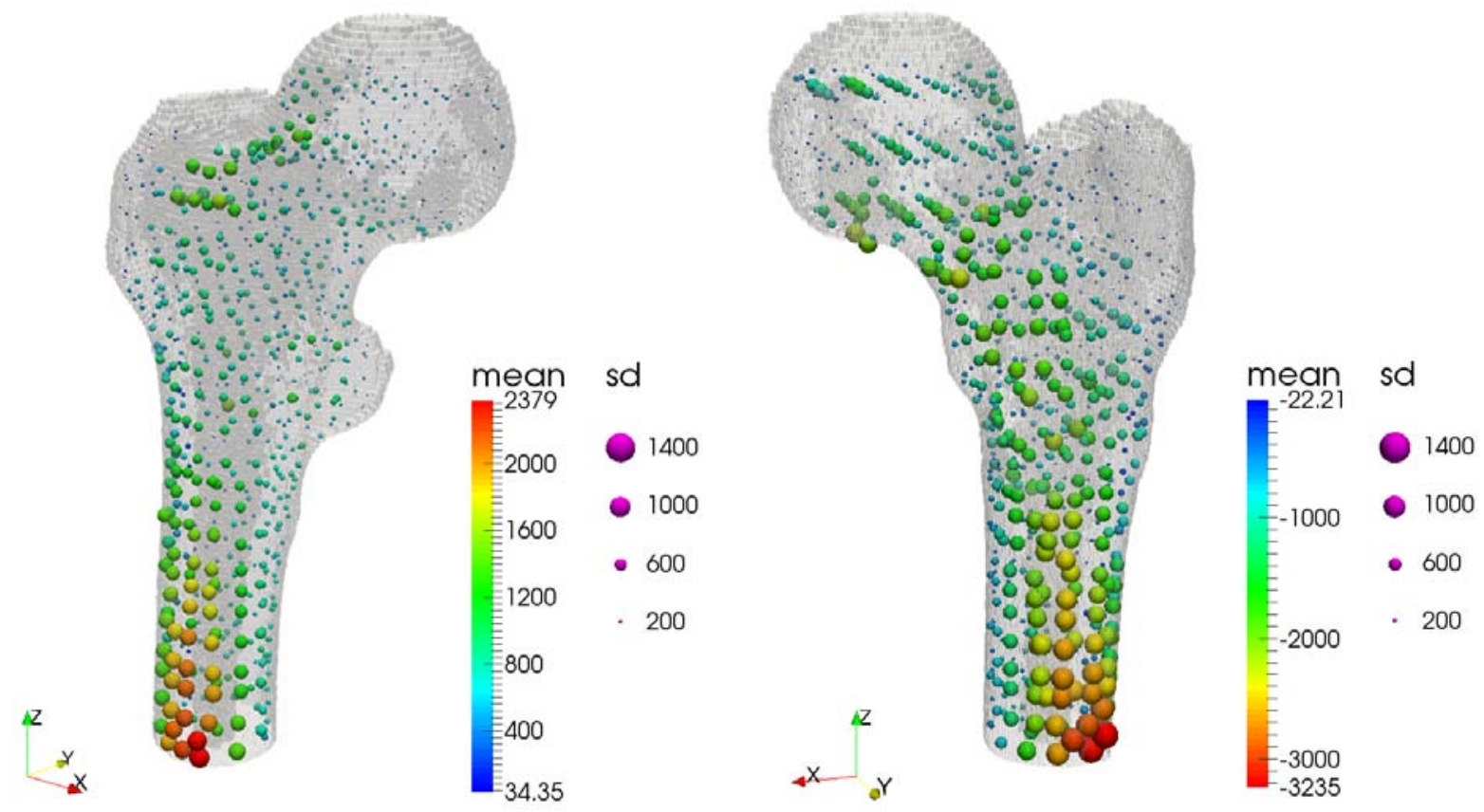

(a) Maximum principal strain $\epsilon_{1}[\mu \mathrm{m} / \mathrm{m}]$, antero- (b) Minimum principal strain $\epsilon_{3}[\mu \mathrm{m} / \mathrm{m}]$, posteromedial view medial view

Figure 3: Mean and standard deviation of principal strains represented by spheres (color $=$ mean, radius of sphere $=$ standard deviation). All 884 post-processing locations are shown at once (554 along the femoral cortex, 330 within the trabecular compartment and the diaphysis). Femur's geometry is indicated by a translucent voxel representation that was derived from the $\mathrm{CT}$ data. For animations of these results the reader is referred to the supplementary material. 


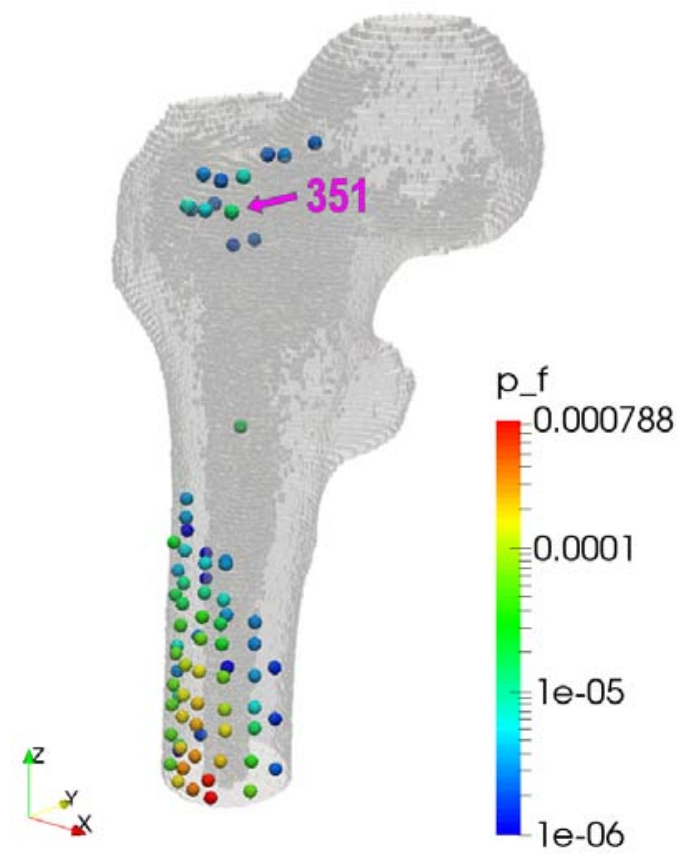

(a) Tensile failure, antero-medial view

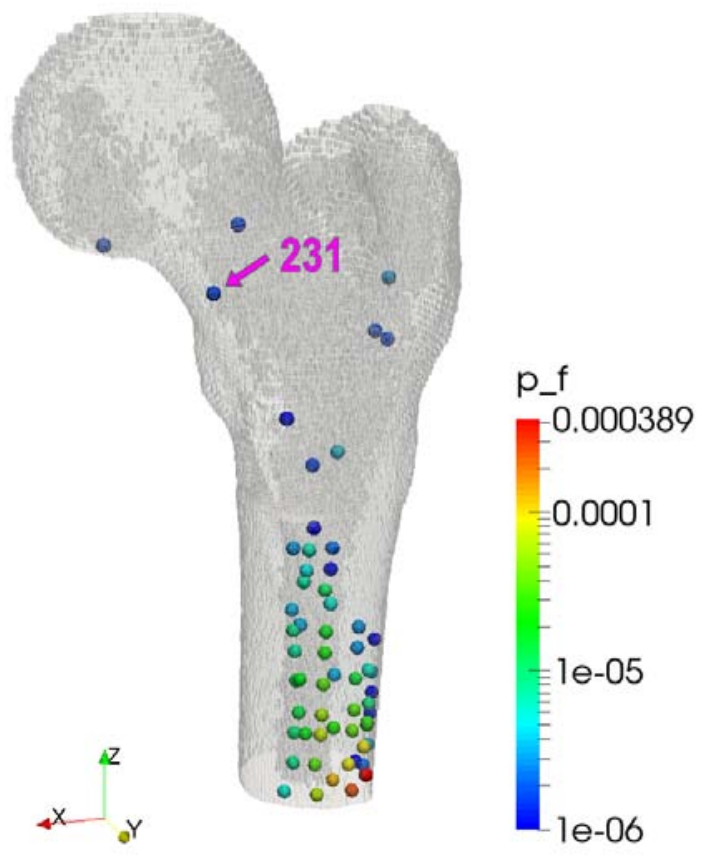

(b) Compression failure, postero-medial view

Figure 4: Probability of failure $p_{f}$ represented as colored unit sphere at locations with $p_{f}>10^{-6}$. Within the femoral neck the largest probability of tensile failure is at point 351 and for compression failure at point 231. Femur's geometry is indicated by a translucent voxel representation that was derived from the CT data. For animations of these results the reader is referred to the supplementary material. 